beruht, ist anzunehmen, wurde aber bislang nicht bewiesen, besonders nicht in In-vivo-Versuchen

Weitere Untersuchungen dieser neuartigen Klasse von Endiin-Chinoniminen zielen auf deren Anwendung in der Totalsynthese von Dynemicin A und von dessen Analoga sowie auf die Zugänglichkeit dieser Verbindungen für die Entwicklung pharmazeutisch einsetzbarer Wirkstoffe.

Eingegangen am 19. August, veränderte Fassung am 10. Oktober 1994 [Z 7252]

[1] M. Konishi, H. Ohkuma, K. Matsumoto, T. Tsuno, H. Kamei, T. Miyaki, T. Oki, H. Kawaguchi, G. D. VanDuyne, J. Clardy, J. Antibiot. 1989, 42, $1449 ;$ M. Konishi, H. Ohkuma, T. Tsuno, T. Oki, G. D. VanDuyne, J. Clardy, J. Am. Chem. Soc. $1990,112,3715$.

[2] Y. Sugiura, T. Shiraki, M. Konishi, T. Oki, Proc. Natl. Acad. Sci. USA 1990, 87, 3831 .

[3] a) Eine hervorragende Übersicht über die Chemie und die Biologie von EndiinAntibiotica geben K. C. Nicolaou, W.-M. Dai, Angew. Chem. 1991, 103, 1453; Angew. Chem. Int. Ed. Engl. 1991, 30, 1387; b) K. C. Nicolaou, W.-M. Dai, S.-C. Tsay, V. A. Estevez, W. Wrasildo, Science 1992, 256,1387 ; c) P. A. Wender, C. K. Zercher, S. Beckham, E.-M. Haubold, J. Org. Chem. 1993, 58 , 5867.

[4] J. Taunton, J. L. Wood, S. L. Schreiber, J. Am. Chem. Soc. 1993, 115, 10378, zit. Lit.

[5] a) M. F. Semmelhack, J. Gallagher, D. Cohen, Tetrahedron Lett. 1990, 31, 1521; b) Y. Sugiura, T. Shiraki, M. Konishi, T. Oki, Proc. Natl. Acad. Sci. USA 1990, 87, 3831 .

[6] R. R. Jones, R. G. Bergman, J. Am. Chem. Soc. 1972, 94, 660; R. G. Bergman, Acc. Chem. Res. 1973, 6, 25.

[7] Für eine Diskussion der intercalierenden Eigenschaften yon Adriamycin siehe a) F. Arcamone in Doxorubicin Anticancer Antibiotics, Academic, New York, 1981; b) S. Neidle, L. H. Pearl, J. V. Skelly, Biochem. J. 1987, 243, 1 ; c) A. Wang, G. Ughetto, G. Quigley, A. Rich, Biochemistry 1987, 26, 1152.

[8] T. Yoon, M. D. Shair, S. J. Danishefsky, G. K. Schulte, J. Org. Chem. 1994, 59 , 3752.

[9] M. D. Shair, T. Yoon, S. J. Danishefsky, J. Org. Chem. 1994, 59, 3755.

[10] a) S. A. Hitchcock, S. H. Boyer, M. Y. Chu-Moyer, S. H. Olson, S. J. Danishefsky, Angew. Chem. 1994, 106, 928; Angew. Chem. Int. Ed. Engl. 1994, 33, 858; b) G. Kim, M. Y. Chu-Moycr, S. J. Danishefsky, J. Am. Chem. Soc. 1990, 112, 2003.

[11] T. Yoon, Dissertation, Yale University, 1994.

[12] H. Kunz, C. Unverzagt, Angew. Chem. 1984, 96, 426; Angew. Chem. Int. Ed. Engl. 1984, 23, 436.

[13] Das Isobenzofuran 13 wurde wie erforderlich in situ hergestellt: a) R. N. Warrener, J. Org. Chem. 1971, 93, 2346; G. M. Priestly, R. N. Warrener, Tetrahedron Lett. 1971, 4295.

[14] Das 2.5:1-Verhältnis der Produkte hat seine Ursache vermutlich in einer endof exo-statt in einer Seitendifferenzierung. Zwar erscheint es vernünftig, daß die Cycloaddition auf der dem Endiin entgegengesetzten Seite stattfindet, doch fehlt bislang der Beweis.

[15] mAMSA ist ein bekannter DNA-Topoisomerase-II-Inhibitor: N. Osheroff, A. H. Coebett, M. J. Robinson, Biochemistry 1993, 32, 3638.

[16] Der Tumor $\left(2 \times 10^{\circ}\right.$ Zellen) wurde subcutan geimpft. Die Behandlung begann am vierten Tag; je drei Mäuse wurden bei jeder Dosis und zur Kontrolle verwendet. Die Tumorgröße wurde nach sieben Tagen nach Beginn der Behandlung bestimmt.

\section{Doppelt lithiierte Oligosilane: Synthese und Struktur des ersten vicinalen Dilithiodisilans**}

Johannes Belzner*, Uwe Dehnert und Dietmar Stalke*

Eine gängige Methode zur Darstellung metallierter Silane ist die Spaltung von Silicium-Silicium- $\sigma$-Bindungen durch Alkalimetalle; diese Reaktion läuft aber nur dann zufriedenstellend $a b$, wenn die Siliciumzentren wenigstens einen aromatischen Substituenten tragen ${ }^{[1]}$. Analoges gilt für die Umsetzung von cyclischen Silanen mit Metallen: Während peralkylierte Cyclosilane von Alkalimetallen lediglich bis zur Stufe der Radikalanionen reduziert werden ${ }^{[2]}$, reagieren arylsubstituierte Silacyclen unter Bindungsspaltung zu präparativ nützlichen difunktionellen $\alpha, \omega$-Dimetallio-Oligosilanen. Vor allem die eingehend untersuchte Umsetzung von Octaphenylcyclotetrasilan und Decaphenylcyclopentasilan mit Lithium wurde zum Aufbau zahlreicher siliciumorganischer Verbindungen genutzt ${ }^{[3]}$. Über analoge Reaktionen von Cyclotrisilanen mit Metallen wurde unseres Wissens bisher nicht berichtet. Diese Lücke wird nun durch die Resultate der Umsetzung des Cyclotrisilans I mit Lithiummetall geschlossen, die, je nach Reaktionsbedingungen, zum 1,3-Dilithiotrisilan 2a oder zum 1,2-Dilithiodisilan 3a führt.

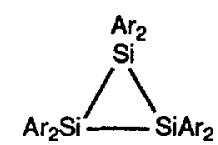

1

$\mathrm{Ar}=2-\left(\mathrm{Me}_{2} \mathrm{NCH}_{2}\right) \mathrm{C}_{6} \mathrm{H}_{4}$<smiles>[2H][Si]([Ge])[Si][Ge]</smiles>

2<smiles>[R][SiH]([R])[SiH2]</smiles>

3
Setzt man das Cyclotrisilan 1 mit zwei Äquivalenten Lithium in 1,4-Dioxan um, so lassen sich rote, extrem luftempfindliche Kristalle der Verbindung $\mathbf{2 a}$ isolieren, deren Protonierung mit Cyclopentadien glatt das Trisilan 2 b liefert. Dic stark konzentrations- und temperaturabhängigen ${ }^{1} \mathrm{H}$ - und ${ }^{13} \mathrm{C}-\mathrm{NMR}$-Spektren von 2 a tragen wenig zur Strukturaufklärung bei, da sie vor allem sehr breite, teilweise stark überlagerte Signale aufweisen. Im ${ }^{29} \mathrm{Si}-\mathrm{NMR}$-Spektrum erscheinen zwei, ebenfalls stark verbreiterte Signale bei $\delta=-26.5$ und -31.8 . Die Identifizierung des Reaktionsproduktes als 1,3-Dilithiotrisilan 2 a basiert deshalb im wesentlichen auf dem Ergebnis der Einkristall-Röntgenstrukturanalyse $^{[4]}$.

Wie die Struktur von 2 a (Abb. 1) im Kristall zeigt, erweist sich auch hier das Konzept der intramolekularen ,Seitenarmkomplexierung ${ }^{\text {" }}$, das bereits bei Organokupferverbindungen ${ }^{[8]}$ und Verbindungen mit hochkoordinierten Siliciumzentren ${ }^{[9]}$ erfolgreich eingesetzt wurde, als besonders fruchtbar. So werden in $\mathbf{2}$ a die Silicium-gebundenen Lithiumatome durch beide Ami-

[*] Dr, J. Belzner, Dipl.-Chem. U. Dehnert

Institut für Organische Chemie der Universität

Tammannstraße 2, D-37077 Götingen

Telefax: Int. + 551/39-9475

Priv.-Doz. Dr. D. Stalke

Institut für Anorganische Chemie der Universität

Tammannstraße 4, D-37077 Göttingen

Telefax: Int. + 551/39-2582

[**] Diese Arbeit wurde von der Deutschen Forschungsgemeinschaft und vom Fonds der Chemischen Industrie gefördert. Wir danken Herrn Prof. M. Rabinovitz für hilfreiche Diskussionen und den Herren R. Machinek, Dr. G. Elter und W. Zolke für die Unterstützung bei den NMR-spektroskopischen Untersuchungen. 


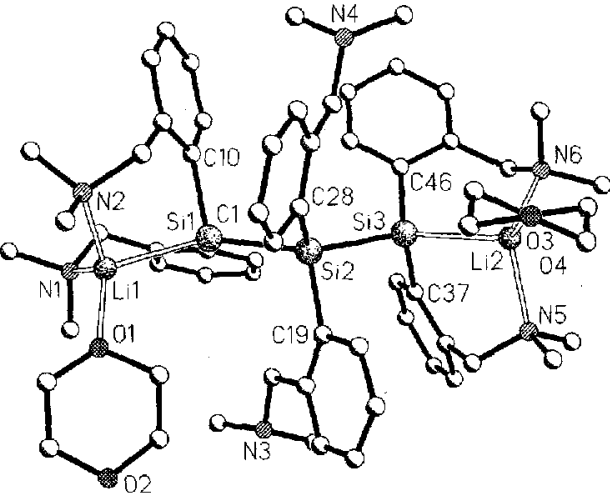

Abb. 1. Struktur yon 2a im Kristall. Drei nicht koordinierte Dioxanmoleküle sind der Übersichtlichkeit halber nicht abgebildet. Ausgewählte. Bindungslängen [pm] und Winkel ["]: Si1-Si2 240.7(3), Si2-Si3 240.1(3), Si1-Li1 255(1), Si3-Li2 254(1) Li1-N1 214(1), Lit-N2 215(1), Li1-O1 197(1), Li2-N5 216(1), Li2-N6 211(1), Li2 O3 199(1); C1-Si1-C10 99.5(3), C19-Si2-C28 101.9(3), C37-Si3-C46 99.8(3), Si1Si2-Si3 136.9(1), Li1-Si1-Si2 131.6(3), Si2-Si3-Li2 129.7(3).

nogruppen der benachbarten $\mathrm{Me}_{2} \mathrm{NCH}_{2} \mathrm{C}_{6} \mathrm{H}_{4}$-Substituenten unter zweifacher Chelatbildung koordiniert. Der mittlere Li-NAbstand beträgt $214 \mathrm{pm}$ und ist damit signifikant länger als der Li-N-Abstand des Lithiumatoms zu Stickstoff-Donorbasen in Kontaktionenpaaren ${ }^{[10]}$. Darüber hinaus koordiniert jeweils ein Dioxanmolekül über ein Sauerstoffatom an jedes Lithiumatom.

Der mittlere Si-Li-Abstand ist mit 254.3 pm deutlich kürzer als in allen bislang strukturell untersuchten Molekülverbindungen mit Si-Li-Kontakten $\left(\left[\mathrm{Me}_{3} \mathrm{SiLi}_{6}{ }^{[11]} 265 \mathrm{pm}\right.\right.$; $\left[\mathrm{Me}_{3} \mathrm{SiLi}(\text { tmeda })_{1.5}\right]^{[12]} 270 \mathrm{pm}$ (tmeda $=N, N, N^{\prime}, N^{\prime}$-Tetramethylethylendiamin); $\left[\left(\mathrm{Me}_{3} \mathrm{Si}\right)_{3} \mathrm{SiLi}(\mathrm{dme})_{1.5}\right]^{[13]} \quad 263 \mathrm{pm}$ (dme = Dimethoxyethan); $\left[\left\{(\mathrm{thf})_{3} \mathrm{Li}\right\}_{2}\left(\mathrm{SiPh}_{2}\right)_{4}\right]^{[14]} 271 \mathrm{pm}$; $\left[\left(\mathrm{Me}_{3} \mathrm{Si}\right)_{3} \mathrm{SiLi}(\text { thf })_{3}\right]^{[15]} 264$ bzw. $267 \mathrm{pm} ;\left[\mathrm{Ph}_{3} \mathrm{SiLi}(\text { thf })_{3}\right]^{[16]}$ $267 \mathrm{pm})$. Durch den sterischen Anspruch der sechs 2$\left(\mathrm{Me}_{2} \mathrm{NCH}_{2}\right) \mathrm{C}_{6} \mathrm{H}_{4}$-Gruppen ist das $\mathrm{LiSi}_{3} \mathrm{Li}$-Rückgrat deutlich gestreckt und nimmt eine annähernd antiperiplanare Konformation ein. Hierbei sind die Bindungswinkel entlang der Li$\mathrm{Si}_{3} \mathrm{Li}$-Kette erheblich größer als der Tetraederwinkel; der zentrale Si-Si-Si-Winkel ist sogar auf $136.9^{\circ}$ aufgeweitet.

$\mathrm{Zu}$ einem anderen Produkt führt die Reaktion von $1 \mathrm{mit}$ Lithium im Überschuß in THF: Sie liefert, ebenso wie die Umsetzung von 2 a mit Lithium unter diesen Bedingungen, das erste 1,2-Dilithiodisilan 3a in Form tiefroter Kristalle. Die Einkristall-Röntgenstrukturanalyse (Abb. 2) zeigt, daß hier, ebenso wie in $\mathbf{2} \mathbf{a}$, beide Lithiumatome an die Siliciumzentren koordiniert sind.

3a kristallisiert mit zwei unabhängigen Molekülen in der asymmetrischen Einheit, die sich nur geringfügig durch die Orientierung der Substituenten unterscheiden (die Strukturparameter des zweiten, nicht abgebildeten Moleküls werden für äquivalente Größen durch einen Schrägstrich getrennt angegeben). Das auf den ersten Blick vielleicht augenfälligste Merkmal ist die unterschiedliche Umgebung der beiden Lithiumatome: Während $\mathrm{Li1}$ von zwei $\mathrm{Me}_{2} \mathrm{NCH}_{2}$-Seitenarmen (mittlerer $\mathrm{Li}-\mathrm{N}$ Abstand $215.2 / 215.8 \mathrm{pm}$ ) und einem thf-Molekül koordiniert wird, ist Li2 von nur einer Aminogruppe (Li-N 212.8/213.7 pm) und zwei thf-Molekülen umgeben. Die beiden Si-Li-Abstände sind untereinander nicht gleich (Si1-Li1 $254.2 / 255.0 \mathrm{pm}$ und Si2-Li2 258.9/259.0 pm). Offensichtlich führt die Koordination durch zwei Amin-Seitenarme zu einem engeren Si-Li-Kontakt (vgl. $254.3 \mathrm{pm}$ in 2a). Die zentrale Si-Si-Bindung zeigt mit 238.0/237.7 pm den Wert einer typischen Silicium-Silicium-Einfachbindung, der nahezu identisch ist mit dem Abstand im Disilan $3 b\left(236.4 / 238.6 \mathrm{pm}^{[17]}\right)$.

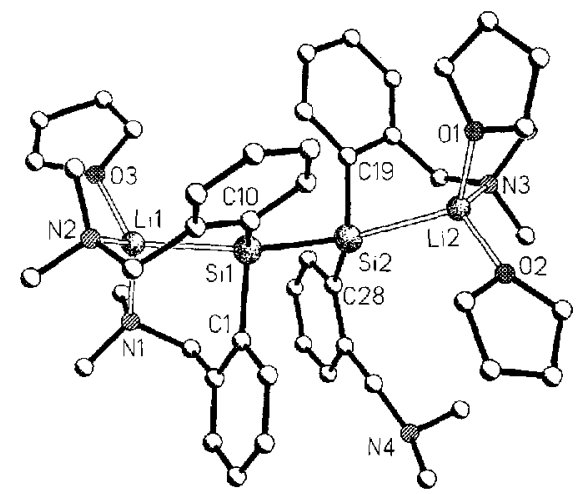

Abb. 2. Struktur von 3a im Kristall. Ausgewählte Bindungslängen [pm] und Win kel $\left[{ }^{C}\right]$ - die durch Schrägstriche abgetrennten Werte bezeichnen jeweils chemisch äquivalente Strukturparameter des zweiten, kristallographisch unabhängigen Moleküls: Si1-Si2 238.0(2)/237.7(2), Si1-Li1 254.2(8)/255.0(8), Si2-Li2 258.9(8) 259.0(8), Li1-O3 195(1)/196(1), Li1-N1 217(1)/215(1), Li1-N2 213(1)/215(1), Li2O1 198(1)/199(1), Li2-O2 196(1)/197(1), Li2-N3 213(1)/214(1); C1-Si1-C10 $100.7(2) / 99.6(2), \mathrm{C} 19-\mathrm{Si} 2-\mathrm{C} 28102.0(2) / 101.3(2)$, Li1-Si1-Si2 143.7(2)/143.7(2), Si1Si2-Li2 $134.9(2) / 134.7(2)$.

Eine Side-on-Koordination der Lithiumatome an die zentrale Element-Element-Bindung ${ }^{[18]}$ wie im Falle der C-C-Einheit in substituierten Dilithioethanen wird in $\mathbf{3 a}$, vermutlich bedingt durch die intramolekulare Komplexierung, nicht gefunden.

In THF als Lösungsmittel wird im ${ }^{29} \mathrm{Si}-\mathrm{NMR}$-Spektrum von $3 \mathbf{a}$ ein $1: 1: 1: 1$-Quartett bei $\delta=-32.8$ beobachtet $^{[19]}$. Die daraus ermittelte ${ }^{29} \mathrm{Si}-{ }^{7} \mathrm{Li}$-Kopplungskonstante von $36 \mathrm{~Hz}$ spricht für den partiell kovalenten Charakter der Si-Li-Bindung ${ }^{[20]}$. Jedoch spiegelt sich die im Vergleich zu anderen lithiierten Siliciumverbindungen kürzere Si-Li-Bindungslänge nicht in einer vergrößerten Kopplungskonstanten wider $\left(\left[\mathrm{Mc}_{2} \mathrm{PhSiLi}\right.\right.$ (thf $\left.)_{x}\right]^{[21]} 51 \mathrm{~Hz} ;\left[\mathrm{Ph}_{3} \mathrm{SiLi}(\text { thf })_{3}\right]^{[161} 45 \mathrm{~Hz} ;\left[\left(\mathrm{Me}_{3} \mathrm{Si}\right)_{3} \mathrm{SiLi}(\text { thf })_{3}\right]$ $37.2 \mathrm{~Hz}^{[15]} ;\left[\left\{(\text { thf })_{3} \mathrm{Li}_{2}\left(\mathrm{SiPh}_{2}\right)_{4}\right]^{[14]} 32.8,40\right.$ und $42 \mathrm{~Hz}$ ).

Diese Resultate zeigen, daß das Cyclotrisilan 1 nicht nur ein wertvolles Syntheseäquivalent für Silandiyle $\mathrm{Ar}_{2} \mathrm{Si}$ : ist ${ }^{[22]}$; seine Umsetzung mit Lithium eröffnet auch den Zugang zu den Dilithioverbindungen $\mathbf{2 a}$ und $\mathbf{3 a}$, die sich ihrerseits als Reagentien zur Übertragung von $\mathrm{Si}_{3}$ - bzw. $\mathrm{Si}_{2}$-Einheiten eignen sollten. Untersuchungen zur Reaktivität dieser Verbindungen sind zur Zeit im Gange.

\section{Experimentelles}

Alle Arbeiten wurden unter Ausschluß von Sauerstoff und Wasser durchgeführt. Bei der Zuordnung der ${ }^{1} \mathrm{H}-{ }^{13} \mathrm{C}$ - und ${ }^{29} \mathrm{Si}-\mathrm{NMR}$-Verschiebungen zu Atomen der terminalen und mittleren Diarylsilandiyl-Einheiten in $\mathbf{2} \mathbf{a}$ und $\mathbf{2} \mathbf{b}$ werden den Elementsymbolen, soweit dies gesichert möglich ist, die Bezeichnungen $(1,3)$ bzw. (2) nachgestellt. Die ${ }^{7} \mathrm{Li}-\mathrm{NMR}$-Spektren wurden extern gegen $\mathrm{LiCl} / \mathrm{H}_{2} \mathrm{O}$ geeicht.

2a: Eine Lösung von $1.99 \mathrm{~g}(2.24 \mathrm{mmol}) 1$ in $28 \mathrm{~mL}$ Dioxan wird mit $31 \mathrm{mg}$ (4.47 mmol) Lithiumspänen versetzt und $22 \mathrm{~h}$ bei $25^{\circ} \mathrm{C}$ gerührt. Die klare rote Lösung wird auf etwa $1 / 3$ ihres Volumens eingeengt, mit $8 \mathrm{~mL}$ Hexan versetzt und durch Filtration von Lithiumresten befreit. Kristallisation bei $4^{\circ} \mathrm{C}$ liefert $1.18 \mathrm{~g}$ ( $49 \%$, Schmp. $50-60^{\circ} \mathrm{C}$ (Zers.)) 2 a als hellrote Kristalle, die nach Trocknung im Hochvakuum noch 2 Äquiv. Dioxan pro Formeleinheit enthalten. ${ }^{1} \mathrm{H}-\mathrm{NMR}$ $\left(250 \mathrm{MHz},\left[\mathrm{D}_{8}\right] \mathrm{THF}, 25^{\circ} \mathrm{C}\right.$ ): $\delta=1.35$ (brs, $12 \mathrm{H} ; \mathrm{NMe}_{2}$ ), 1.93 (s, $24 \mathrm{H} ; \mathrm{NMe}_{2}$ ), $2.3-3.4$ (brs, $8 \mathrm{H} ; \mathrm{CH}_{2}$ ), 3.6-4.1 (brs, $4 \mathrm{H} ; \mathrm{CH}_{2}$ ), 6.4-6.9 (brs, $\left.10 \mathrm{H}\right), 6.91$ (t, $\left.{ }^{3} J=7.5 \mathrm{~Hz}, 4 \mathrm{H}\right), 7.18\left(\mathrm{~d},{ }^{3} J=7.2 \mathrm{~Hz}, 4 \mathrm{H}\right), 7.6-8.1$ (brs, $\left.4 \mathrm{H} ; 6-\mathrm{H}(1,3)\right), 8.6-8.9$ (brs, $2 \mathrm{H} ; 3-\mathrm{H}(2)) ;{ }^{7} \mathrm{Li}-\mathrm{NMR}\left(155.45 \mathrm{MHz}\right.$, THF $\left./ \mathrm{C}_{6} \mathrm{D}_{6}(5: 1), 25^{\circ} \mathrm{C}\right): \delta=0.90(\mathrm{~s})$; ${ }^{29} \mathrm{Si}-\mathrm{NMR}\left(99.3 \mathrm{MHz},\left[\mathrm{D}_{8}\right] \mathrm{THF}, 25^{\circ} \mathrm{C}\right): \delta=-26.5(\mathrm{Si}-2),-31.8(\mathrm{Si}-1,3)$

2b: Eine auf $-78^{\circ} \mathrm{C}$ gekühlte Lösung von $1.05 \mathrm{~g}(0.93 \mathrm{mmol}) 2 \mathrm{a}$, das noch 2.5 Aquiv. Dioxan pro Formeleinheit enthielt, in $10 \mathrm{~mL}$ THF wird mit $0.19 \mathrm{~mL}$ (2.33 mmol) Cyclopentadien versetzt und unter Rühren auf Raumtemperatur erwärmt. Nach dem Austausch des Lösungsmittels gegen $15 \mathrm{~mL}$ Hexan und Abfiltrieren von ungelösten Bestandteilen scheiden sich aus dem eingeengten Filtrat $0.56 \mathrm{~g}$ $\left(68 \%\right.$, Schmp. $\left.171-172^{\circ} \mathrm{C}\right) \mathbf{2 b}$ in Form hydrolyseempfindlicher Kristalle ab. ${ }^{1} \mathrm{H}-\mathrm{NMR}\left(250 \mathrm{MHz}, \mathrm{C}_{6} \mathrm{D}_{6}\right): \delta=1.79\left(\mathrm{~s}, 12 \mathrm{H} ; \mathrm{NMe}_{2}\right), 2.00\left(\mathrm{~s}, 24 \mathrm{H} ; \mathrm{NMe}_{2}\right), 3.04$ (s, $\left.4 \mathrm{H} ; \mathrm{CH}_{2}\right), 3.25,3.27$ (AB-System, ${ }^{2} \mathrm{~J}=14 \mathrm{~Hz}, 8 \mathrm{H} ; \mathrm{CH}_{2}$ ), 5.87 (s, ${ }^{1} J(\mathrm{Si}, \mathrm{H})=200.5 \mathrm{~Hz}, 2 \mathrm{H}$; SiH), $6.92\left(\mathrm{dd},{ }^{3} J(4,5)=7.5 \mathrm{~Hz},{ }^{3} J(5,6)=7.2 \mathrm{~Hz}, 4 \mathrm{H}\right.$; 
$5-\mathrm{H}(1,3)), 7.09\left(\mathrm{dd},{ }^{3} J(3,4)=7.4 \mathrm{~Hz},{ }^{3} J(4,5)=7.5 \mathrm{~Hz}, 4 \mathrm{H} ; 4-\mathrm{H}(1,3)\right), 7.22(\mathrm{dd}$ $\left.{ }^{3} J(4,5)=7.5 \mathrm{~Hz},{ }^{3} J(5,6)=7.2 \mathrm{~Hz}, 2 \mathrm{H} ; 5-\mathrm{H}(2)\right), 7.33\left(\mathrm{dd},{ }^{3} J(3,4)=7.6 \mathrm{~Hz}\right.$, $\left.{ }^{3} J(4,5)=7.5 \mathrm{~Hz}, 2 \mathrm{H} ; 4-\mathrm{H}(2)\right), 7.36\left(\mathrm{~d},{ }^{3} J(3,4)=7.4 \mathrm{~Hz}, 4 \mathrm{H} ; 3-\mathrm{H}(1,3)\right), 7.71(\mathrm{~d}$ $\left.{ }^{3} J(5,6)=7.2 \mathrm{~Hz}, 4 \mathrm{H} ; 6-\mathrm{H}(1,3)\right), 7.81\left(\mathrm{~d},{ }^{3} J(3,4)=7.6 \mathrm{~Hz}, 2 \mathrm{H} ; 3-\mathrm{H}(2)\right), 8.60$ (d, $\left.{ }^{3} J(5,6)=7.2 \mathrm{~Hz}, 2 \mathrm{H} ; 6-\mathrm{H}(2)\right) ;{ }^{13} \mathrm{C}-\mathrm{NMR}\left(62.9 \mathrm{MHz}, \mathrm{C}_{6} \mathrm{D}_{6}\right): \delta=45.15,45.28$ $\left(2 \times \mathrm{NMe}_{2}\right), 63.99,64.46\left(2 \times \mathrm{CH}_{2}\right), 125.98,128.22,128.98,129.05,129.50$, $(5 \times \mathrm{CH}), 135.54,135.74(\mathrm{C}-1(1,2,3)), 138.43,138.72(\mathrm{C}-6(1,2,3)), 146.04(\mathrm{C}-$ 2(1.3)), $148.07(\mathrm{C}-2(2)) ;{ }^{29} \mathrm{Si}-\mathrm{NMR}\left(59.6 \mathrm{MHz}, \mathrm{C}_{6} \mathrm{D}_{6}\right): \delta=-37.5$ (brs), -43.9 $\left(\mathrm{d},{ }^{1} J(\mathrm{Si}, \mathrm{H})=203 \mathrm{~Hz}\right)$; $\mathrm{MS}(70 \mathrm{eV}) m / z(\%): 890\left(M^{+},<1 \%\right), 297\left(\mathrm{Ar}_{2} \mathrm{SiH}^{+}\right.$, 100)

3a: Eine Lösung von $1.09 \mathrm{~g}(1.23 \mathrm{mmol}) 1$ in $18 \mathrm{~mL}$ THF wird mit $45 \mathrm{mg}$ $(7.38 \mathrm{mmol})$ Lithiumspänen versetzt und $3 \mathrm{~d}$ bei $25^{\circ} \mathrm{C}$ gerührt. Die klare, tiefrote Lösung wird durch Filtration von Lithiumresten befreit, auf etwa $3 \mathrm{~mL}$ eingeengt und mit $10 \mathrm{~mL}$ Pentan versetzt. Kristallisation bei $-15^{\circ} \mathrm{C}$ liefert $0.87 \mathrm{~g}(53 \%$, Schmp. $45-50^{\circ} \mathrm{C}$ (Zers.)) 3a in Form dunkelroter Kristalle, die pro Formeleinheit noch 4 Aqquiv. THF enthalten. ${ }^{1} \mathrm{H}-\mathrm{NMR}\left(250 \mathrm{MHz}, \mathrm{C}_{6} \mathrm{D}_{6}\right): \delta=1.82$ (brs, $24 \mathrm{H}$; $\left.\mathrm{NMe}_{2}\right), 2.7-3.4\left(\mathrm{brs}, 8 \mathrm{H} ; \mathrm{CH}_{2}\right), 6.8-7.1(\mathrm{~m}, 4 \mathrm{H} ; 3-\mathrm{H}), 7.0-7.3(\mathrm{~m}, 8 \mathrm{H} ; 4,5-\mathrm{H})$. 8.36 (brs, $4 \mathrm{H} ; 6-\mathrm{H}) ;{ }^{13} \mathrm{C}-\mathrm{NMR}\left(75.5 \mathrm{MHz}, \mathrm{C}_{6} \mathrm{D}_{6}\right): \delta=46.14\left(\mathrm{NMe}_{2}\right), 67.71$ $\left(\mathrm{CH}_{2}\right), 122.44,126.08(\mathrm{C}-4,5), 130.23(\mathrm{C}-3), 139.12(\mathrm{C}-6), 142.48(\mathrm{C}-2), 162.20$ (C-1); ${ }^{7} \mathrm{Li}-\mathrm{NMR}\left(155.45 \mathrm{MHz}, \mathrm{C}_{6} \mathrm{D}_{6}\right): \delta=1.36$ (s); ${ }^{29} \mathrm{Si}-\mathrm{NMR}(59.6 \mathrm{MHz}, \mathrm{THF} /$ $\left.\mathrm{C}_{6} \mathrm{D}_{6}(5: 1), 25^{\circ} \mathrm{C}\right): \delta=-32.8\left({ }^{1} /(\mathrm{Si}, \mathrm{Li})=36 \mathrm{~Hz}\right)$.

Eingegangen am 9. August 1994 [Z 7224]

[1] A. G. Brook, H. Gilman, J. Am. Chem. Soc. 1954, 76, 278- 279; b) H. Gilman, G. D. Lichtenwalter. ibid. 1958, $80,608-611$.

[2] B. Kirste, R. West, H. Kurreck, J. Am. Chem. Soc. 1985, 107, 3013-3016.

[3] a) H. Gilman, D. J. Peterson, A. W. Jarvie, H. J. S. Winkler, J. Am. Chem. Soc 1960, 82, 2076; b) H. Gilman, G. L. Schwebke, ibid. 1963, 85, 1016; c) E. Hengge, D. Wolfer, J. Organomet. Chem. 1974, 66, 413-424.

[4] Kristalldaten von 2a: $\mathrm{Ar}_{6} \mathrm{Si}_{3} \mathrm{Li}_{2}$ (dioxan $)_{2}+3$ Dioxan $\left(\mathrm{Ar}=2-\left(\mathrm{Me}_{2} \mathrm{NCH}_{2}\right)\right.$ $\left.\mathrm{C}_{6} \mathrm{H}_{4}\right), M=1424.05$, triklin, Raumgruppe $P \overline{1}, a=1442.6(3), b=1633.1(3)$, $c=2025.1(4) \mathrm{pm}, \quad \alpha=66.87(3), \quad \beta=79.54(3), \quad \gamma=69.96(3)^{\circ}, \quad V=$ $4.116(2) \mathrm{nm}^{3}, Z=2, \quad \rho_{0 \mathrm{cos}}=1.149 \mathrm{gcm}^{-3}, \quad F(000)=1548, \lambda=71.073 \mathrm{pm}$, $T=-120^{\circ} \mathrm{C}, \quad \mu\left(\mathrm{Mo}_{\mathrm{K}}\right)=0.113 \mathrm{~mm}^{-1}, \quad$ Kristallabmessungen: $0.6 \times 0.4 \times$ $0.4 \mathrm{~mm}, 8^{\circ} \leq 2 \theta \leq 45^{\circ}$; von 12625 gesammelten Reflexen sind 10698 unabhängig, die alle zur Verfeinerung von 952 Parametern mit Hilfe von 212 Restraints für die 1-2- und 1-3-Abstände der koordinierten und unkoordinierten Dioxanmoleküle benuizt wurden; größte Restelektronendichte: $581 \mathrm{enm}^{-3}$ $R 1(F>4 \sigma(F))=0.057$ und $w R 2=0.161$ (alle Daten) mit $R 1=\sum\left\|F_{\mathrm{n}}|-| F_{\mathrm{c}}\right\| /$ $\sum\left|F_{0}\right|$ and $w R 2=\left(\sum w\left(F_{0}^{2}-F_{c}^{2}\right)^{2} / \sum w\left(F_{0}^{2}\right)^{2}\right)^{0.5}-$ Kristalldaten von 3a $\mathrm{Ar}_{4} \mathrm{Si}_{2} \mathrm{Li}_{2}(\mathrm{thf})_{3}\left(\mathrm{AT}=2-\left(\mathrm{Me}_{2} \mathrm{NCH}_{2}\right) \mathrm{C}_{6} \mathrm{H}_{4}\right), M=823.16$, triklin, Raumgruppe $P \overline{1}, \quad a=1052.5(2), \quad b=2155.3(4), \quad c=2376.2(5) \mathrm{pm}, \quad \alpha=66.26(3)$ $\beta=89.48(3), \gamma=88.30(3)^{\circ}, V=4.932(2) \mathrm{nm}^{3}, Z=4, \rho_{\text {ber }}=1.109 \mathrm{~g} \mathrm{~cm}^{-3}$ $F(000)=1784, \lambda=71.073 \mathrm{pm}, T=-120^{\circ} \mathrm{C}, \mu\left(\mathrm{Mo}_{\mathrm{K} \alpha}\right)=0.113 \mathrm{~mm}^{-1}$, Kri stallabmessungen: $0.7 \times 0.6 \times 0.6 \mathrm{~mm}, 4^{\circ} \leq 2 \theta \leq 45^{\circ}$; von den 12732 gesammelten Reflexen sind 12663 unabhängig, die alle zur Verfeinerung von 1099 Parametern benutzt wurden; größte Restelektronendichte: $470 \mathrm{enm}^{-3}$ $R 1(F>4 \sigma(F))=0.080$ und $w R 2=0.242$ (alle Daten). Die Daten beider Strukturen wurden auf einem Stoe-AED-Diffraktometer gesammelt. Die Intensitätsmessungen wurden an schockgekühiten Kristallen im Öltropfen ${ }^{[s]}$ nach der $20 /(\omega)$-Abtastung durchgeführt. Die Strukturen wurden durch Direkte Methoden gelöst (SHELXS-90) [6] und nach dem Kleinste-FehlerquadrateVerfahren gegen $F^{2}$ verfeinert [7]. Weitere Einzelheiten zu den Kristallstrukturuntersuchungen können beim Direktor des Cambridge Crystallographic Data Centre, 12 Union Road, GB-Cambridge CB2 1EZ, unter Angabe des vollständigen Literaturzitats angefordert werden.

[5] T. Kottke, D. Stalke, J. Appl. Crystallogr. 1993, 26, 615-619.

[6] G. M. Sheidrick, Acta Crystallogr. Sect. A 1990, 46, 467-473.

[7] G. M. Sheldrick, SHELXL-93, Programm zur Kristallstrukturverfeinerung, Göttingen, 1993

[8] a) G. van Koten, J. G. Noltes in Comprehensive Organometallic Chemistry (Hrsg.: G. Wilkinson, F. G. A. Stone, E. W. Abel), Pergamon Press, Oxford, 1982, S. 709-763; b) G. van Koten, J. Organomet. Chem. 1990, 400, 283-301.

[9] a) R. J. P. Corriu, C. Guerin, Adv. Organomet. Chem. 1982, 20, 265-312; b) C. Chuit, R. J. P. Corriu, C. Reye, J. C. Young, Chem. Rev. 1993, 93, 1371-1448.

[10] a) K. Gregory, P, von R. Schleyer, R. Snaith, Adv. Inorg. Chem. 1991, 37 , 47-142; b) H. Gornitzka, D. Stalke, Organometallics, im Druck; c) U. Olsher, R. M. Izatt, J. S. Bradshaw, N. K. Dalley, Chem. Rev. 1991, 91, 137-164.

[11] a) T. F. Schaaf, W. Butler. M. D. Glick, J. P. Oliver, J. Am. Chem. Soc. 1974, 96 7593 7594; b) W. H. Ilsley, T. F. Schaaf, M. D. Glick, J. P. Oliver, ibid. 1980 $102,3769-3774$

[12] B. Teclé, W. H. Ilsley, J. P. Oliver, Organometallics 1982, 1, 875-877.

[13] G. Becker, H.-M. Hartmann, A. Münch, H. Riffel, Z. Anorg. Allg. Chem. 1985 , $530,29-42$.

[14] G. Becker, H.-M. Hartmann, E. Hengge, F. Schrank, Z. Anorg. Allg. Chem. $1989,572,63-74$.

[15] A. Heine, R. Herbst-Irmer, G. M. Sheldrick, D. Stalke, Inorg. Chem. 1993, 32 $2694-2698$.

[16] H. V. R. Dias, M. M. Olmstead, K. Ruhlandt-Senge, P. P. Power, J. Organomet. Chem. 1993, $462,1-6$.
[17] J. Belzner, N. Detomi, H. Ihmels, M. Noltemeyer, Angew. Chem. 1994, 106, 1949-1950; Angew: Chem. Int. Ed. Engl. 1994, 33, 1854-1855.

[18] a) M. Walczak, G. D. Stucky, J. Organomet. Chem. 1975, 97, 313-323; b) M. Walczak, G. Stucky, J. Am. Chem. Soc. 1976, 98, 5531-5539; c) A. Sekiguchi, T. Nakanishi, C. Kabuto, H. Sakurai, ibid. 1989, 111, 3748-3750; d) Chem. Lett. 1992, 867-870.

[19] Versuche, die in Kristallen von 3a beobachtete, unterschiedliche Umgebung der Li-Atome auch in wenig koordinierenden Lösungsmitteln wie $\left[\mathrm{D}_{8}\right]$ Toluo oder $\mathrm{C}_{6} \mathrm{D}_{6}$ NMR-spek troskopisch nachzuweisen, waren erfolglos.

[20] C. Lambert, P. von R. Schleyer, Angew. Chem. 1994, 106, 1187-1199; Angew. Chem. Int. Ed. Engl. 1994, 33, 1129-1140.

[21] U. Edlung, T. Lejon, T. K. Venkatachalam, E. Buncel, J. Am. Chem. Soc. 1985 107, 6408-6409.

[22] Siehe beispielsweise J. Belzner, H. Ihmels, Tetrahedron Lett. 1993, 34, 6541 6544.

\section{„Antennen-Effekt" in multichromophoren Cyclodextrinen **}

\section{Ludovic Jullien, Josette Canceill, Bernard Valeur*,} Elisabeth Bardez und Jean-Marie Lehn*

In Analogie zu Antennen-Pigmenten von PhotosyntheseEinheiten ${ }^{[1]}$ besteht eine photochemische Antenne aus einer Ansammlung von Chromophoren, die aufgrund eines großen Gesamtquerschnitts für die Lichtabsorption eine hohe Photonensammeleffizienz haben und die Anregungsenergie von den Chromophoren - direkt oder nach Transfer von Chromophor zu Chromophor - auf einen Energieacceptor übertragen, der die Umwandlung der absorbierten Lichtenergie ermöglicht. Dieser Acceptor kann eine lichtemittierende Spezies sein, z.B. ein Lanthanoid-Ion oder ein organischer Fluorophor, die das einfallende Licht in solches höherer Wellenlänge umwandelt. Ein Beispiel hierfür bieten die Komplexe von Lanthanoid-Ionen mit Polypyridinliganden ${ }^{[2]}$, die vielversprechende Fluoreszenzmarker für chemische und biochemische Zwecke sind. Weitere photochemische molekulare Funktionseinheiten, die auf Antennen basieren, können entworfen werden ${ }^{[3]}$, wobei mehrkernige Metallkomplexe besondere Aufmerksamkeit erlangten ${ }^{[4]}$. Der ,Antennen-Effekt" ist auch bei lichtsammelnden Polymeren, deren Ketten in Intervallen mit Chromophoren substituiert wurden, untersucht worden ${ }^{[5,6]}$. Bei den früher beschriebenen multichromophoren $\beta$-Cyclodextrinen $(\beta-C D)$ ist die Position der Chromophore im Raum weitaus besser definiert, woraus sich deutliche Vorteile für das Verständnis des Energietransferprozesses ergeben ${ }^{[7,8]}$. Bei Arbeiten an diesen Systemen haben wir ein neues multichromophores $\beta$-CD synthetisiert, um den Antennen-Effekt, wie er in Photosyntheseeinheiten auftritt, nachzuahmen, und wir berichten hier über den sehr effizienten Energietransfer von sieben 6-Carboxy-2-naphthyl-Antennenchromophoren auf ein in den Hohlraum eingeschlossenes Merocyanin, DCM-OH (Abb, 1).

[*] Prof. Dr. B, Valeur, Dr. E. Bardez

Laboratoire de Chimie Générale (CNRS ER 77)

Conservatoire National des Arts et Métiers

292 rue Saint-Martin, F-75003 Paris (Frankrcich)

Telefax: Int. +1/40272362

Prof. Dr. J.-M. Lehn, Dr. L. Jullien, Dr. J. Canceill

Chimie des Interactions Moléculaires (CNRS UPR 285)

Collège de France

11 Place Marcelin Berthelot, F-75005 Paris (Frankreich)

Telefax: Int. + 1/44271356

[**] Diese Arbeit wurde vom Centre National de la Recherche Scientifique (CNRS) gefördert. Die Autoren danken Dr. R. Pansu, Ecole Normale Supérieure, Cachan, für seine Hilfe bei den Fluoreszenzabklingexperimenten. 